\title{
IDENTIFICAÇÃO DOS PROBLEMAS RELACIONADOS A MEDICAMENTOS ANTIMICROBIANOS EM PACIENTES INTERNADOS NA CLÍNICA MÉDICA DE UM HOSPITAL ESCOLA
}

\author{
Geisllen Cristina da Silva Paes ${ }^{1 *}$, Carlos Eduardo Faria Ferreira ${ }^{2}$ \\ ${ }^{1}$ Acadêmica do Curso de Farmácia FMC - Faculdade de Medicina de Campos. \\ ${ }^{2}$ Orientador, Coordenador e Professor titular do Curso de Farmácia FMC - Faculdade de Medicina de Campos \\ * Email: geisllencristina@windowslive.com
}

\section{RESUMO}

$\mathrm{O}$ uso indiscriminado de medicamentos antimicrobianos pode acarretar graves consequências como reações adversas para os pacientes, o aumento da resistência bacteriana e para o hospital gastos desnecessários. A fragmentação da responsabilidade do tratamento farmacológico, especialmente devido à presença de múltiplos prescritores de medicamentos para um mesmo paciente, juntamente com a extensiva quantidade de medicamentos novos e o elevado grau de morbidade e mortalidade associadas aos medicamentos, são, em grande parte, responsáveis pelos problemas relacionados ao uso de medicamentos. Os Problemas Relacionados aos Medicamentos Antimicrobianos são reações adversas ao medicamento, este considerado inevitável. Já os erros na medicação como prescrição incorreta, tempo de tratamento inadequado, erros de dispensação, omissão ou erros na administração estes por sua vez são considerados evitáveis podendo ou não causar danos à saúde do paciente. O objetivo do estudo é identificar problemas relacionados a medicamentos antimicrobianos. Foram estudados 32 pacientes, e encontradas 7 interações medicamentosas, erros de omissão e reação adversa, sendo que $25 \%$ dos pacientes adquiriram infecção hospitalar. Com o presente trabalho verifica-se que os antimicrobianos são uma classe cuja prescrição é frequente na clínica médica, apresentando quantidade de interações medicamentosas significativas, reação adversa e erros de omissão.

Palavras- chave: Antimicrobianos, Problemas

Relacionados a Medicamentos.

\begin{abstract}
The indiscriminate use of antimicrobial drugs can have serious consequences as adverse reactions for patients, increased bacterial resistance and for hospital expenditures unnecessary. The fragmentation of the responsibility for pharmacological treatment, especially due to the presence of multiple prescription drugs for the same patient, together with an extensive amount of new drugs and high degree of morbidity and mortality associated with the drugs, are, to a large extent, related to Use of medications. The problems related to Antimicrobial drugs are adverse reactions to the medication, this is considered inevitable and errors in medicine such as incorrect prescription, treatment time, dispensing errors, omission or mistakes in administration are in turn avoidable concepts that may or may not cause harm to the Health of the patient. The aim of the study is to identify problems related to antimicrobial drugs. 32 patients were studied, 7 drug interactions, errors of omission and adverse reaction were found, $25 \%$ of the patients acquiring nosocomial infection. The present study shows that antimicrobials are a class whose prescription is frequent in the medical clinic, presenting a number of significant drug interactions, an adverse reaction and errors of omission.
\end{abstract}

Keywords: Antimicrobials, Drug Related Problems. 


\section{INTRODUÇÃO}

Desde que foram descobertos os antibióticos tem sido de suma importância para saúde mundial, representando grande avanço na medicina. Esses medicamentos trouxeram a esperança e a possibilidade da cura de doenças que antes levavam muitas pessoas a morte (OLIVEIRA, 2006).

Por outro lado, o uso indiscriminado de medicamentos antimicrobianos pode acarretar graves consequências como reações adversas para os pacientes, o aumento da resistência bacteriana e para o hospital gastos desnecessários.

Em muitos casos, os antibióticos são utilizados sem que haja a real necessidade, sem indicação médica, doses inadequadas e por tempo prolongado ou insuficiente. As consequências são resistência bacteriana e sua proliferação. Em hospitais caracterizam-se como a principal causa de infecções hospitalares (OLIVEIRA, 2006).

Segundo Oliveira (2006), como resultado das práticas inadequadas realizadas pelos profissionais de saúde, destacando o emprego não racional de antibióticos em pacientes internados, o hospital está se tornando reservatório de bactérias resistentes e uma via de transmissão de graves doenças.

A fragmentação da responsabilidade do tratamento farmacológico, especialmente devido à presença de múltiplos prescritores de medicamentos para um mesmo paciente, juntamente com a extensiva quantidade de medicamentos novos e o elevado grau de morbidade e mortalidade associadas aos medicamentos, são, em grande parte, responsáveis pelos problemas relacionados ao uso de medicamentos (POLISEL; BERGÊ, 2014).

Segundo Polisel e Bergê, (2014) cerca de $30 \%$ dos pacientes hospitalizados utilizam antibióticos, o que representa aproximadamente $1 / 3$ dos gastos hospitalares com medicamentos e $20 \%$ a $50 \%$ da receita hospitalar. No Brasil, a incidência de infecção nosocomial em Unidade de Terapia Intensiva varia entre $5 \%$ e $10 \%$.

Diversas estratégias tem sido implementadas na área da utilização e monitorização do uso de medicamentos, destacam-se os estudos de avaliação da prescrição médica. A prescrição é um compromisso entre as estruturas sanitárias e seus usuários e uma instrução do prescritor para o profissional que a recebe, sendo de fundamental importância que as informações nela contidas sejam claras e adequadas, de modo a evitar dispensações equivocadas e consequentemente contribuir para o uso inadequado dos medicamentos. (POLISEL; BERGÊ, 2014).

Os Problemas Relacionados aos Medicamentos Antimicrobianos são reações adversas ao medicamento, este considerado inevitável e erros na medicação como prescrição incorreta, tempo de tratamento inadequado, erros de dispensação, omissão ou erros na administração estes por sua vez são considerados evitáveis podendo ou não causar danos à saúde do paciente (COMITÊ DE CONSENSO, 2002).

A principal consequência da terapêutica incorreta de antibióticos é a indução à resistência bacteriana, o que acarreta em graves infecções, principalmente a nível hospitalar (OLIVEIRA, 2006).

Com a redução do uso inadequado de antibióticos ocorre não só a redução do número de infecções hospitalares, mas também gera economia para o hospital, pois estes medicamentos, na maioria das vezes, possuem preços muito elevados.

O objetivo do presente estudo é identificar os principais Problemas Relacionados a Medicamentos Antimicrobianos no setor de internação da clínica médica em um hospital escola.

\section{MATERIAL E MÉTODOS}

O estudo foi realizado no setor de Clínica Médica de um Hospital Escola, situado no norte do Estado do Rio de Janeiro. Trata-se de um Hospital Filantrópico, mantido por uma Fundação também filantrópica, uma entidade jurídica de direito privado, de domínio público, sem fins lucrativos, com sede e foro na Cidade de Campos dos Goytacazes, Estado do Rio de Janeiro, Brasil. O Hospital conta com diferentes ambulatórios em várias especialidades como Angiologia, Cardiologia (inclusive pediátrica), Cirurgia Geral, Cirurgia Oncológica, Cirurgia Plástica, Dermatologia, Infectologia, entre outras. Além disso, realiza também diferentes tipos de exames como cateterismos, eletrocardiogramas e tomografias, além de vários exames laboratoriais como Citopatologia, Sorologia e vários outros exames dentro das Análises Clínicas. O setor de Clínica Médica atende em média a 80 (oitenta) internações e 250 (duzenetos e cinquenta) consultas ambulatoriais mensais. A clínica médica conta com 12 leitos masculinos e 12 femininos, sendo 8 leitos reservados para o atendimento oncológico. $\mathrm{O}$ estudo foi realizado a partir de 32 pacientes que utilizaram medicamentos antimicrobianos durante o período de internação. O grupo selecionado foi atendido e participaram da pesquisa pelo Projeto 
Conciliação ${ }^{1}$ no período de Março e Abril de 2017. Os dados foram obtidos por meio de formulários preenchidos com base nas informações dos prontuários de cada paciente do referido Projeto além das justificativas de solicitações de antimicrobianos enviadas a Farmácia.

Foram avaliadas as seguintes variáveis: Medicamento Antimicrobiano, tempo de tratamento, tipo de infecção e Problemas relacionados a medicamentos. $\mathrm{O}$ trabalho foi realizado a partir da submissão ao Comitê de Ética sob o número de aprovação 997.220 do Projeto Conciliação.

A análise dos dados foi obtida com os programas Microsoft Word e Microsoft Excel.

\section{RESULTADOS E DISCUSSÃO}

Foram estudados 32 pacientes, destes 17 são do sexo feminino e 15 do sexo masculino de diferentes faixas etárias (Tabela 1).

Observou-se que a faixa etária acima de 50 anos, apresentou maior indicação para terapia antimicrobiana decorrente do estado imunológico e co-morbidades associadas. Não foi encontrada diferença significativa entre o uso desses fármacos entre os gêneros.

Na Figura 1 observa-se que a maioria dos pacientes utilizou apenas 2 medicamentos antimicrobianos e 1 paciente 5 medicamentos antimicrobianos.

\begin{tabular}{cc}
\hline Idade & Número de Pacientes \\
\hline Abaixo dos 20 & 2 \\
20 a 30 & 1 \\
30 a 40 & 4 \\
40 a 50 & 4 \\
50 a 60 & 8 \\
60 a 70 & 3 \\
70 a 80 & 4 \\
80 a 90 & 4 \\
Acima dos 90 & 2 \\
\hline Total & 32 \\
\hline
\end{tabular}

Tabela 1. Relação de Idades dos pacientes estudados.

De acordo com Carneiro et al. (2011,) a associação antimicrobiana está relacio-nada com falsa proteção. O uso simultâneo de fármacos é recomendado em situações específicas, a fim de aumentar o espectro antimicrobiano. Entretanto, quando empregados de maneira inadequada, associam-se a um risco de toxicida-de, seleção de patógenos resistentes, além de aumentar os custos institucionais.

\section{Número de Antimicrobianos utilizados por cada paciente}

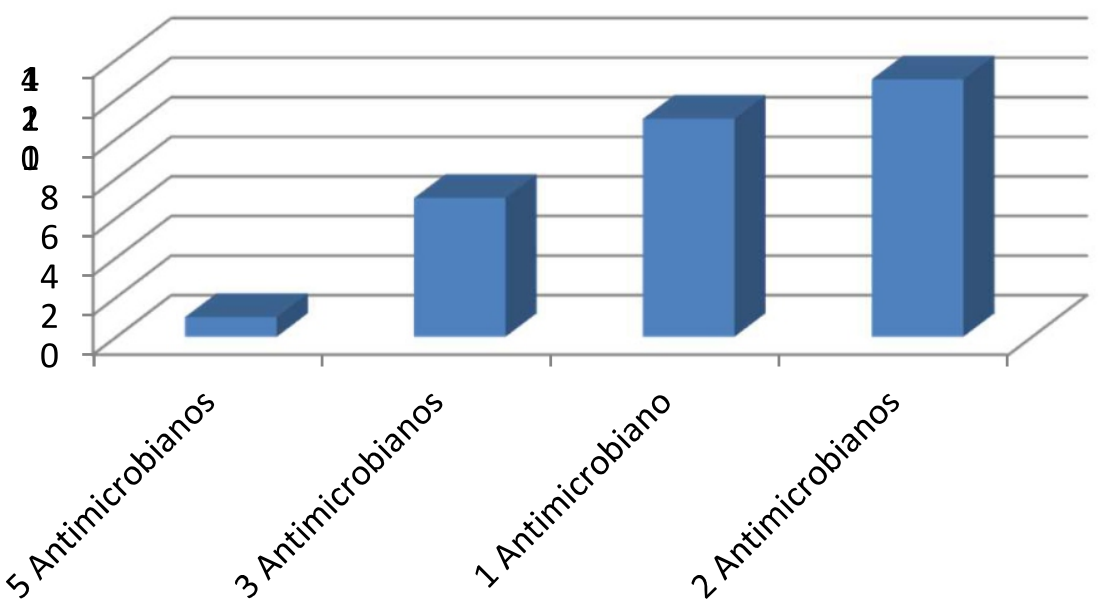

Figura 1 - Número de Antimicrobianos utilizados por cada paciente durante o período de internação. 


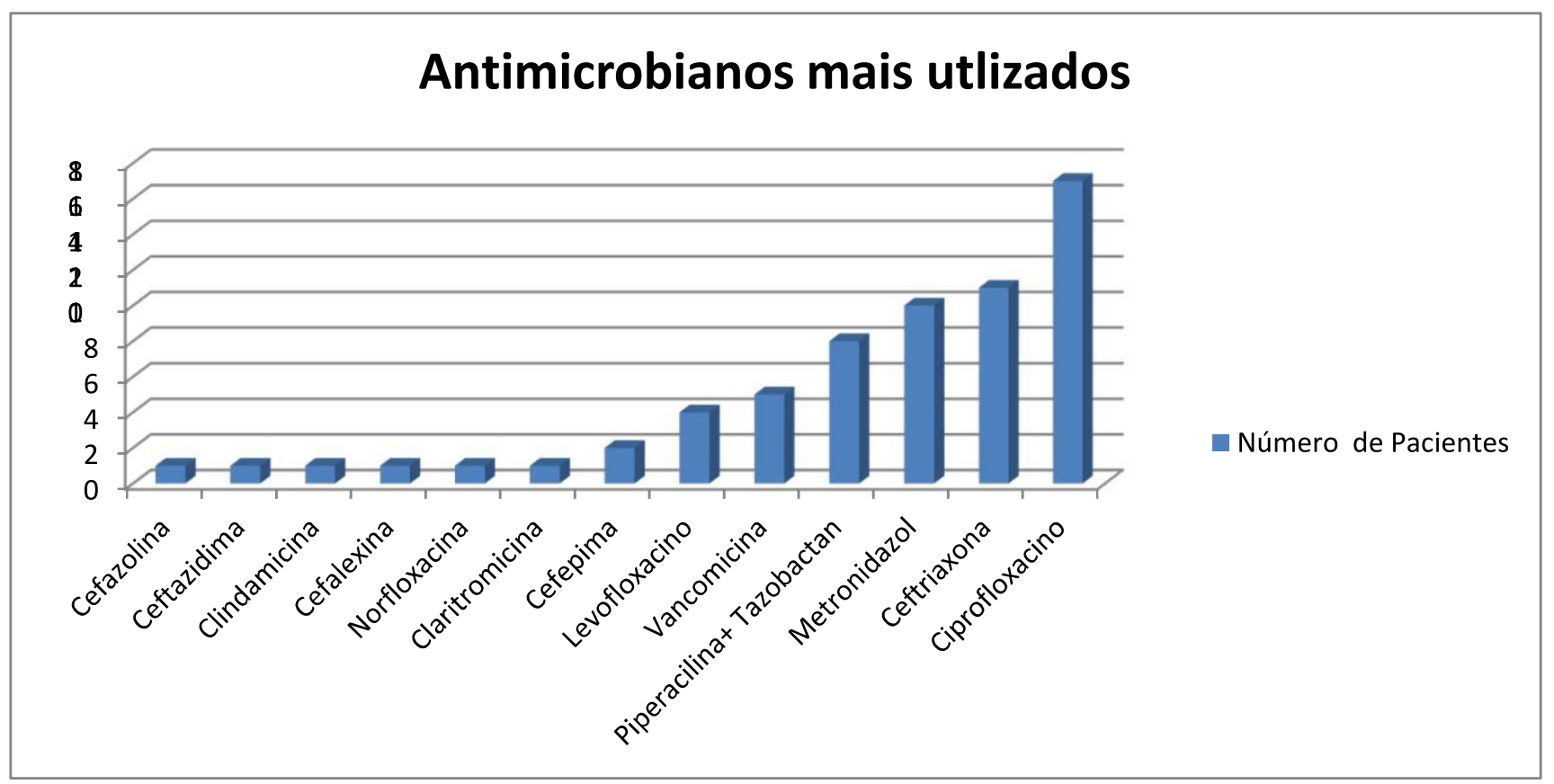

Figura 2. Medicamentos antimicrobianos mais utilizados durante o período de estudo.

Na Figura 2 observam-se os medicamentos antimicrobianos mais utilizados. Ciprofloxacino foi o medicamento mais utilizado para tratamento de infecções como: Infecções do trato urinário, Infecção abdominal, abcesso perianal, pós-operatório com infecção de desbridamento de mão dentre outros.

O Ciprofloxacino foi o único medicamento que durante os estudos do Projeto Conciliação apresentou interação medicamentosa grave. Segundo o Guia de Interações Medicamentosas da Universidade Federal de Goiás (2011), é importante destacar que essa interação não interfere na efetividade do medicamento, entretanto pode trazer sérios danos a saúde do paciente. As interações foram:

O uso concomitante de Ciprofloxacino com Tramadol aumenta o risco de depressão do Sistema Nervoso Central e Depressão Respiratória. Cinco pacientes faziam uso dessas medicações.

O uso concomitante de Ciprofloxacino com Prednisona aumenta o risco de tendinite e ruptura do tendão. Apenas um paciente fazia uso dessas medicações.

O concomitante de Ciprofloxacino com Dexametasona aumenta o risco de tendinite e ruptura do tendão. Apenas um paciente fazia uso dessas medicações.

Foram observados 4 devoluções de Metronidazol e 1 de Ceftriaxona, porém não foram suspensos pelo médico responsável, caracterizando erro de omissão, os responsáveis pelo setor foram informados. Houve 1 possível reação adversa a Vancomicina, na qual o paciente apresentava diarreia com uso do medicamento. Observou-se muitas variações no tempo de tratamento com mínimo de 3 e máximo de 46 dias.

A Figura 3 apresenta o tipo de infecção, obtidas a partir das justificativas de solicitação médica de antimicrobianos. Em $25 \%$ da amostranão foram encontradas indicações; $41 \%$ dos pacientes foram internados com algum tipo de infecção não adquirida em ambiente hospitalar, 25\% após internação adquiriram algum tipo de infecção hospitalar sendo a mais relatada pneumonia hospitalar e 9\% internados com infecção, porém durante a internação adquiriram infecção hospitalar.

Os fatores desencadeantes de infecções hospitalares podem ser intrínsecos e extrínsecos. O risco intrínseco provém de distúrbios fisiológicos que alteram os mecanismos de defesa da própria pessoa. O risco extrínseco é proveniente do ambiente (OLIVEIRA, 2006).

Devemos considerar importante que a incidência de infecções é o resultado final da qualidade das diversas atividades e profissionais envolvidos na assistência, e não somente a simples relação bactéria-hospedeiro da visão biológica clássica (OLIVEIRA, 2006). 


\section{Tipo de Infecção}

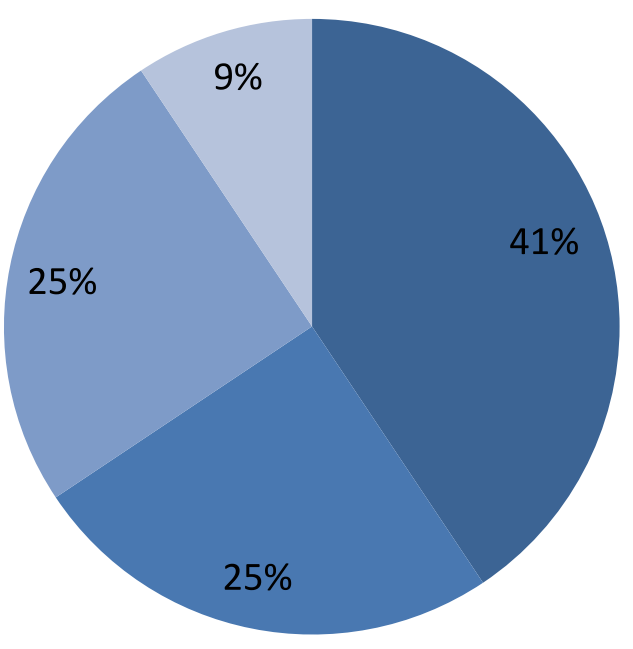

Comunitária

Hospitalar

Não registrada

Comunitária + Hospitalar

Figura 3. Tipo de infecção informações obtidas através de justificativas de solicitação de antimicrobianos.

\section{CONCLUSÃO}

Com o presente trabalho a partir da amostra analisada verifica-se que os antimicrobianos são uma classe cuja prescrição é frequente na clínica médica e que os principais problemas relacionados os antimi-crobianos fora, interações medicamentosas significativas, uma reação adversa e erros de omissão.
Percebe-se a necessidade de mais estudos de utilização de antimicrobianos que analisem prescrições. São fundamentais o desenvolvimento de pesquisa em farmacovigilância, uma vez que esses medicamentos são essenciais para o tratamento das doenças infecciosas e têm uma influência incontestável no aumento de resistência microbiana sendo que seu uso inadequado torna-se nocivo para todos.

\section{REFERÊNCIAS}

ALVIM, M. M. ; SILVA, L. A. da; LEITE, Isabel Cristina Gonçalves and SILVERIO, Marcelo Silva.Eventos adversos por interações medicamentosas potenciais em unidade de terapia intensiva de um hospital de ensino. Rev. bras. ter. intensiva [online]. v.27, n.4, p.353-359, 2015. ISSN 0103-507X. disponível em: <http:// dx.doi.org/10.5935/0103-507X.20150060>. Acesso em: 21 maio. 2017.

CARNEIRO, M. et al.O uso de antimicrobianos em um hospital de ensino: uma breve avaliação.Rev. Assoc. Med. Bras. [online]. v.57, n.4, p.421-424, 2011. ISSN 0104-4230. Disponível em: <http://dx.doi.org/10.1590/ S0104-42302011000400016>. Acesso em: 21 maio. 2017.

COMITÊ DE CONSENSO, 2002. FOLHA Informativa Farmacoterapêutica CINFARMA - Centro de Informação Farmacêutica do Departamento de Farmacovigilância, DNME/MINSA, v. 0/ n. 6/ 7 Abr./ Set. 2015. Disponível em: <http://www.ordemfarmaceuticosangola.org/PDF/Folha\%20Farmacoterapeutica\%20n67.pdf>. Acesso em: 21 maio. 2017.

GUIA DE INTERAÇÕES MEDICAMENTOSAS. Universidade Federal de Goiás, Hospital das

Clínicas, Coordenação de Farmácia. Disponível em ://farmacia.hc.ufg.br/up/734/o/

Guia_de_Interacoes_Medicamentosas.pdf?1409055761. Acesso em 20 de agosto de 2019.

KATZUNG, B.G., MASTERS, S.B., TREVOR, A. J. , Farmacologia Básica e Clínica, $12^{\circ}$ ed AMGH Editora Ltda. 2014, pág 790. 
OLIVEIRA, A.L. Resistência bacteriana a antibióticos: uma análise da conduta hospitalar. Revista Cesumar. Ciências Humanas e Sociais Aplicadas jan./jun. 2006, V. 11, nº 1, pp 59-69.

OLIVEIRA, W. L.; BRANCO, A. B. . Avaliação da antibioticoterapia em pacientes internados no Hospital Regional do Guará - DF. Com. Ciências Saúde. v.18, n.2, p. 107-114, 2007.

POLISEL,C.G. ,BERGÊ, R.S. Avaliação da conformidade de prescrições médicas e dispensação de antimicrobianos. Revista Brasileira Promoção Saúde, Fortaleza, 27(1): 21-28, jan./mar., 2014.

RODRIGUES, F. A. ; BERTOLDI, A. D. .Perfil da utilização de antimicrobianos em um hospital privado. Ciênc. saúde coletiva [online]. v.15, suppl.1, p.1239-1247, 2010. ISSN 1413-8123. Disponível em: <http:// dx.doi.org/10.1590/S1413-81232010000700033>. Acesso em: 21 maio. 2017.

SANTANA, R. S. et al.The cost of excessive postoperative use of antimicrobials: the context of a public hospital. Rev. Col. Bras. Cir. [online].

SILVA, D. G., NORONHA, A. G. G. M., Intervenção farmacêutica na antibioticoterapia hospitalar. Importância clínica e impacto econômico. Infarma, v. 13, n. 3/4, 2001.v.41, n.3, p.149-154, 2014. ISSN 0100-6991.

Disponível em: <http://dx.doi.org/10.1590/S0100-69912014000300003>. Acesso em: 21 maio. 2017. 